\title{
Design of $M$-Band NPR Cosine-Modulated Filterbank Using IFIR Technique
}

\author{
Ram Kumar Soni ${ }^{1}$, Alok Jain ${ }^{2}$, Rajiv Saxena ${ }^{3}$ \\ ${ }^{1}$ Department of Electronics \& Communication Engineering, Samrat Ashok Technological Institute (Polytechnic), Vidisha, India, \\ ${ }^{2}$ Department of Electronics \& Instrumentation Engineering, Samrat Ashok Technological Institute, Vidisha, India, ${ }^{3}$ Department of \\ Electronics \& Communication Engineering, Jaypee University of Engineering \& Technology, Raghogarh, Guna, India. \\ Email: soniram04@rediffmail.com, alokjain6@rediffmail.com,rajiv.saxena@jiet.ac.in
}

Received September $28^{\text {th }}, 2010$, revised November $1^{\text {st }}, 2010$; accepted November $3^{\text {rd }}, 2010$.

\begin{abstract}
This paper presents a design of M-band near perfect reconstructed (NPR) cosine-modulated filterbank (CMFB). The prototype filter is formulated as an interpolated finite impulse response (IFIR) filter. Suitable stretched factor is used to provide considerable reduction in computation cost as well as minimum value of error parameters. Further minimization in errors has been achieved by applying a linear gradient optimization technique. The IFIR approach provides reduction in stopband energy as side-lobe-fall-off-rate (SLFOR) in magnitude response of the prototype filter is improved. Design examples have been included to illustrate the effectiveness of the proposed technique over the existing work.
\end{abstract}

Keywords: $C M F B, I F I R, N P R$, Window

\section{Introduction}

Cosine modulated filterbank find wide applications in many areas of signal processing such as data compression of speech, audio and video signals, denoising, feature detection and extraction and adaptive signal processing [1-5]. In this approach all the analysis filters $H_{k}(z)$ and synthesis filters $F_{k}(z)$ are designed by the cosine modulation of prototype lowpass filter as shown in Figure 1. As a result, the design of filterbank becomes simple, more realizable and the computation cost reduces to the cost of one prototype filter plus modulation overhead. This design technique has been extensively studied by many prominent authors [3-7]. The use of finite impulse response (FIR) filter is oftenly preferred over the infinite impulse response (IIR) filter in the design of prototype lowpass filter. Such type of filter has some desirable features like linear-phase, inherent-stability, negligible-quantization noise, etc. [4]. However, the computational requirements of a FIR filter are usually greater than an IIR filter. This is essentially true in CMFB design for high stopband attenuation and narrow transition band width. The high computational requirement of FIR filter can be reduce by exploiting the redun- dancy in filter coefficients. Neuvo et al. [8] have developed a technique for FIR filter design in which the required specifications are obtained by the cascade of FIR sections. The overall structure is known as interpolated finite impulse response (IFIR) filter. This approach requires less multipliers than the conventional single stage FIR implementation.

In the proposed work, an NPR type cosine modulated filterbank is designed. The required prototype filter is formulated by IFIR technique. The comparison has been made with earlier reported work of Furado et al. [9] and Kha et al. [10]. In first one the frequency response masking (FRM) approach has been used for the design of prototype filter of a filterbank. Similarly, in second case prototype filter is designed using standard FIR approach and a convex optimization technique is used to minimize the associated errors of filterbank. The contribution of this paper is summarized below:

1) The prototype filter is designed using IFIR approach to provide reduction in computation complexity at high stopband attenuation and narrow transition band width.

2) Most popular variable Kaiser window function is used to design the prototype filter. 


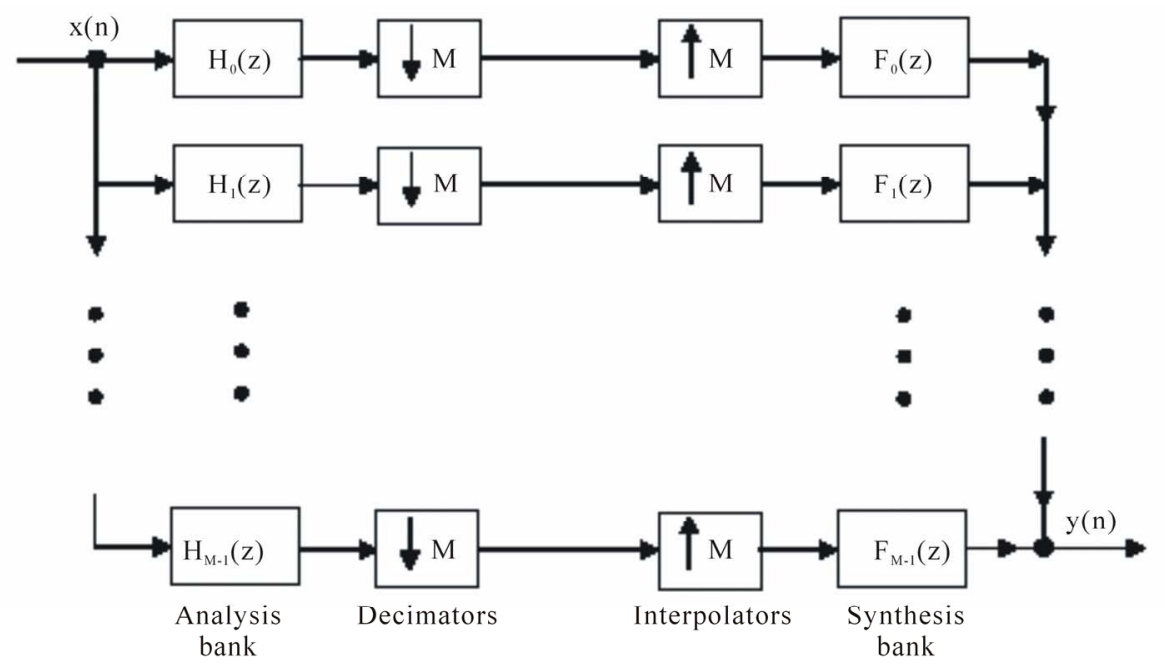

Figure 1. $M$-band cosine modulated filterbank.

3) NPR type cosine modulation approach is used for the design of filterbank. It avoids the computation of large matrix sets of perfect reconstruction (PR) condition and thereby further reduces the computational burden during the implementation.

4) The reconstructed output of NPR system is not exact replica of input due to reconstruction and aliasing error. A linear gradient optimization is applied in which the cutoff frequency is changed in order to approximate the power complementary property.

5) Examples are included to evaluate the performance of the proposed technique in terms of error parameters, computational complexity, group delay and stopband energy.

This paper is organized as follow: The IFIR filter with their performance parameters are described in Section 2. This section is also included the design procedure of prototype lowpass filter using proposed IFIR. Section 3 covered the description of cosine modulation approach for filterbank design and parameters related with computational complexity. The algorithm of linear gradient optimization technique with required objective function is provided in Section 4. Section 5 included design examples of eight, sixteen and thirty two band NPR CMFB. The performance of proposed work with earlier reported publications are discussed in Section 6. Finally concluding remarks are made in Section 7.

\section{Design of Prototype Filter}

The impulse response coefficients of a causal $N^{\text {th }}$-order linear phase FIR filter $p[n]$ using window technique is given by [4]:

$$
p[n]=w[n] h[n]
$$

where, $h[n]$ is the impulse response of the ideal lowpass filter and is expressed as:

$$
h[n]=\frac{\sin \left(\omega_{\mathrm{c}}(n-0.5 N)\right)}{\pi(n-0.5 N)}
$$

where, $\left(\omega_{\mathcal{C}}\right)$ is the cutoff frequency of the ideal lowpass filter and $w[n]$ is the Kaiser window function. For the given value of passband $\left(\omega_{p}\right)$ and stopband $\left(\omega_{s}\right)$ frequencies the filter order is calculated by [4]:

$$
N=\frac{A_{S}-7.95}{14.36(\Delta \omega)}
$$

where, $\Delta \omega=\left(\omega_{s}-\omega_{p}\right) / 2 \pi$ is the normalized transition bandwidth, $A_{\mathrm{s}}$ is the stopband attenuation and $\omega_{c}=\left(\omega_{s}+\omega_{p}\right) / 2$.

In the design of narrowband FIR lowpass filter the filter order is very high consequently the required number of adders and multipliers are also high. Therefore, in proposed work an IFIR technique is used for the design of prototype lowpass filter. This technique is computationally more efficient than standard FIR filter design technique. In IFIR technique the final prototype lowpass filter can be obtained by Eq. (4) [11]

$$
P(z)=G\left(z^{L}\right) I(z)
$$

where, $G\left(z^{L}\right)$ is the up-sampled version of model filter $G(z)$ with the stretching factor of $L$ and $I(\mathrm{z})$ is the interpolator filter. In the proposed work, both filters are designed by using Kaiser window function. Input parameters like stopband attenuation, passband ripple are taken same as desire in final prototype IFIR filter. Only the passband and stopband frequencies are different. For the model filter the values are relaxed by factor of $L$, i.e., 
$\left(L \omega_{p}\right)$ and $\left(L \omega_{s}\right)$, respectively. Similarly, for the interpolator filter the value of passband and stopband frequencies are $\left(\omega_{p}\right)$ and $\left(2 \pi / L-\omega_{s}\right)$, respectively.

The reduction in computation complexity provided by IFIR filter is very much depends upon the stretch factor. There is an optimum value of stretch factor $L$ given in (5), which provides upto $90 \%$ reduction in computation at the cost of other performance parameters [11]. Therefore, the selection of $L$ is depends upon the applications, for which the IFIR filter is designed. The optimum value of $L$ is given as [11].

$$
L_{\text {opt }}=\left[\frac{2 \pi}{\omega_{p}+\omega_{s}+\sqrt{2 \pi\left(\omega_{s}-\omega_{p}\right)}}\right]
$$

The computational complexity is stated in terms of number of multipliers needed for implementation. The number of multiplier in case of linear phase FIR structure, polyphase structure and IFIR structure can be calculated as below $[4,12]$

Multiplier in linear phase FIR structure $=\lceil N / 2\rceil$

$$
\text { Multiplier in polyphase structure }=\left\lceil\frac{N}{2}\right\rceil
$$

$$
\text { Multiplier in IFIR structure }=\left\lceil\frac{\left(N_{m}\right)}{2}+\frac{\left(N_{i}\right)}{2}\right\rceil
$$

Similarly,

$$
\begin{gathered}
\text { Adders in linear phase FIR structure }=\lceil N\rceil \\
\text { Adders in polyphase structure }=\lceil N / 2\rceil \\
\text { Adders in IFIR structure }=\left\lceil N_{m}+\mathrm{N}_{\mathrm{i}}\right\rceil
\end{gathered}
$$

where, $N, N_{m}$ and $N_{i}$ are the filter order for standard FIR filter, model filter and interpolator, respectively. Since, the filter order of model filter and interpolator are less because first one is stretched version of desired prototype filter and second one has wide band and large transition width. Therefore, the multiplier (multi) needed in IFIR filter is much less than standard FIR filter and achieved computational reduction is given as:

$\%$ Computational reduction $=\frac{\left(\text { multi }_{F I R}-\text { multi }_{I F I R}\right)}{\text { multi }_{F I R}}$

A FIR filter exhibits property of linear phase. Therefore, its phase response is constant and derivatives of phase with respect to frequency called the group delay $\left(\tau_{g}\right)$ is given by [12]:

$$
\tau_{g}=\frac{d(\theta \omega)}{d \omega}=\frac{N}{2}
$$

Hence, in linear phase FIR filter the groups delay depends upon filter order. The proposed technique provides improvement in SLFOR, consequently reduction in stopband energy $\left(E_{\mathrm{s}}\right)$. For a lowpass prototype filter stopband energy is given as [13]

$$
E_{s}=\int_{\omega_{s}}^{\pi}|H(\omega)|^{2} d \omega
$$

\section{Cosine Modulation}

Cosine modulation is one of the efficient technique which provides minimum computational cost in filterbank design. In this technique all the filters of synthesis and analysis sections are obtained by cosine modulation of a linear phase lowpass prototype filter $[4,12]$.

$$
\left.\begin{array}{l}
\left.h_{k}(n)=2 p(n) \cos \left[(2 k+1) \frac{\pi}{2 M}\left(n-\frac{N}{2}\right)+(-1)^{k} \frac{\pi}{4}\right]\right) \\
\left.f_{k}(n)=2 p(n)\right) \cos \left[(2 k+1) \frac{\pi}{2 M}\left(n-\frac{N}{2}\right)-(-1)^{k} \frac{\pi}{4}\right]
\end{array}\right\}
$$

$$
\text { for } 0 \leq k \leq M-1 \text {, and } 0 \leq n \leq N
$$

Here, $M$ is the number of bands in filterbank and $h_{k}(n)$ and $f_{k}(n)$ are the impulse responses of the filters of analysis and synthesis sections, respectively. The relation between the reconstructed output $Y(z)$ and input $X(z)$ is expressible in the $\mathrm{z}$-domain as

$$
Y(z)=T_{0}(z) X(z)+\sum_{l=1}^{M-1} T_{l}(z) X\left(z e^{-j 2 \pi l / M}\right)
$$

Here, $T_{0}(z)$ is the overall distortion transfer function and determines the distortion caused by the overall system for the unaliased component $X(z)$ of the input signal and

$$
T_{l}(z)=\sum_{k=0}^{M-1} F_{k}(z) H_{k}\left(z e^{-j 2 \pi l / M}\right)
$$

for $l=1,2, \cdots, M-1 \quad$ is called the aliased transfer function and determine how well the aliased components $X\left(z e^{-j 2 \pi l / M}\right)$ of the input signal are attenuated. To cancel aliasing and achieve PR, it is required that [4]

$$
T_{0}(z)=c z-n_{d}, c \neq 0,
$$

where, $n_{d} \cdots$ is positive integer and

$$
T_{l}(z)=0, \text { for } l=1,2,3, \cdots, M-1
$$

For PR the criteria for the prototype filter are very strict. Fortunately, in many practical applications the PR property can be slightly relaxed, resulting in NPR [4].

\section{Optimization Technique}

In NPR system, the PR conditions are certainly relaxed 
by allowing small amount of error. There are three types of errors occurs at the reconstructed output, viz., amplitude, phase and aliasing [4,5]. The stopband attenuation plays an important role in analysis of these parameters. High stopband attenuation results smaller aliasing error but larger reconstruction error. The peak to peak reconstruction and the peak aliasing errors are define in (19-20) [10]

$$
\begin{gathered}
E_{p p}=\max _{\omega}\left(M T_{0}\left(e^{j \omega}\right)\right)-\min _{\omega}\left(M T_{0}\left(e^{j \omega}\right)\right) \\
E_{a}=\max _{\omega} E\left(e^{j \omega}\right)
\end{gathered}
$$

where, $E\left(e^{j \omega}\right)=\left[\sum_{l=1}^{M-1}\left|T_{l}\left(e^{j \omega}\right)\right|^{2}\right]^{1 / 2}$

In NPR system, the measures of these error parameters are approximately and are given by the following equations:

$$
\begin{gathered}
\qquad\left|P\left(e^{j \omega}\right)\right| \approx 0 \quad \text { for }|\omega|>\pi / M \\
T_{0}\left(e^{j \omega}\right) \approx 1, \\
\text { where } T_{0}\left(e^{j \omega}\right)=\sum_{k=0}^{2 M-1}\left|P\left(e^{j(\omega-k \pi / M)}\right)\right|^{2}
\end{gathered}
$$

The accuracy of the first approximation gives aliasing and accuracy of the second gives the reconstruction error. The phase error can be eliminating completely by using linear phase prototype filter. Other two distortion parameters can be minimized by applying suitable optimization technique. Much of work have been done in this field [5,14-16]. Initially, Johnston [16] developed a nonlinear optimization technique, later on many prominent authors such as Creusere et al. [14] and Lin et al. [15] and others [5] have simplified it and developed single variable linear optimization techniques with different objective functions. In the proposed work, a linear gradient optimization technique is used with objective function given in (23). The cutoff frequency of the model filter is selected as variable parameter to optimize the objective function.

$$
\varphi=\max \|\left|P\left(e^{j \omega}\right)\right|^{2}+P\left(\left.e^{j(\omega-\pi / M)}\right|^{2}-1 \mid\right.
$$

for $0 \leq \omega<\pi / M$

Initially, input parameters, i.e., sampling rate, number of bands, stretch factor, passband and stopband frequencies, passband ripple and stopband attenuation of prototype filter are specified. Based on these input parameters the passband and stopband frequencies of model filter and interpolator filter are calculated. Determine the cutoff frequency, transition band and filter length of model filter as well as interpolator filter. Initialize, different optimization pointers like step size, search direction, flag and initial as well as expected minimum possible values of objective function. Inside the optimization loop, design the model filter, interpolated filter, up-sampled model filter and finally, obtained the prototype lowpass filter using Equation (4). Determine the other bandpass filters of analysis and synthesis sections using cosine modulation. In optimization routine cutoff frequency of model filter is gradually change as per the search direction and calculate the corresponding value of objective function. Algorithm comes out from the loop as it attained the minimum value of objective function and obtained the optimized value of reconstruction error.

The flowchart of the optimization algorithm is given in appendix and implemented on MATLAB 7.0 on Pentium IV processor.

\section{Design Examples}

In this section, the performance evaluation of the proposed technique has been done with the help of design examples. The performance of the filterbank is examined in terms of stopband attenuation, reconstruction error, aliasing error, computational complexity, group delay and stopband energy in used prototype filter. The effectiveness of proposed work is compared with the earlier reported work for same input parameters.

Example 1: An eight-band cosine modulated filterbank has been designed for same specifications as given by Kha et al. [10] with stopband attenuation $\left(A_{\mathrm{s}}\right)=$ $35.8 \mathrm{~dB}$, stopband frequency $\left(\omega_{s}\right)=0.12 \pi$. Figure 2(a), shows the frequency response of the prototype filter designed by standard FIR technique. In this proposed work, the prototype filter is designed using IFIR technique. For the given design parameters of prototype filter, the required model, up-sampled model and interpolator filters are obtained for stretched factor $L=2$. The stopband attenuation and passband ripple for these filters are same as desire in prototype filter, however the stopband frequency is different. For model and interpolator filters these values are $\left(L \omega_{s}\right)=0.24 \pi$ and $\left(2 \pi / L-\omega_{s}\right)=$ $0.88 \pi$, respectively. The obtained magnitude responses of these filters are shown in Figure 2(b). The magnitude response of the resultant IFIR filter is shown in Figure 2(c). Filter coefficients are optimized by the proposed gradient optimization algorithm (given in Appendix) to reduce the reconstruction error. The optimized eightband CMFB is shown in Figure 2(d). The obtained values of reconstruction error, aliasing error, group delay, stopband energy and the required number of multipliers and adders in case of Kha et al. [10] and in proposed IFIR technique are given in Table-1. The magnitude responses of reconstruction and aliasing errors are shown 
in Figures 3(a) and 3(b), respectively.

Example 2: In this example, sixteen-band cosine modulated filterbank is designed using same specifications as given in [10]. The stopband attenuation and stopband frequency are taken as same, i.e., $\left(A_{s}\right)=45 \mathrm{~dB}$, $\left(\omega_{s}\right)=0.0590 \pi$. The stopband attenuation for model and interpolator are same as in prototype filter, i.e., $45 \mathrm{~dB}$. The stopband frequency for these filters are $\left(L \omega_{s}\right)=$ $0.118 \pi$ and $\left(2 \pi / L-\omega_{s}\right)=0.941 \pi$, respectively. The magnitude response of prototype IFIR filter is shown in Figure 4(a) and the magnitude responses of sixteen band CMFB is shown in Figure 4(b). The optimized value of reconstruction error, aliasing error and reduction in computation cost, stopband energy and group delay are given in Table 1. The reconstruction error and aliasing error are shown in Figure 4(c) and 4(d), respectively.

Example 3: A thirty two-band cosine modulated filterbank has been designed for same specifications as given by Kha et al.[10] with stopband attenuation $\left(\mathrm{A}_{\mathrm{s}}\right)=$

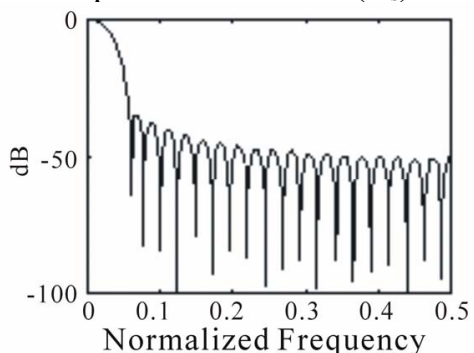

(a)

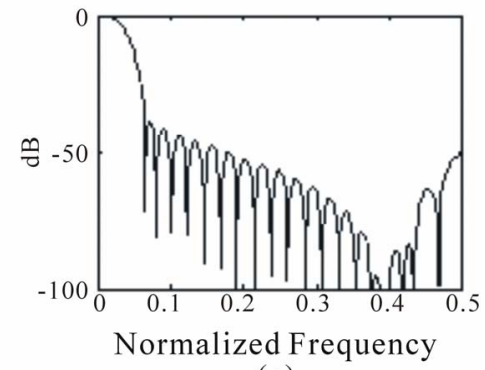

(c)
$100 \mathrm{~dB}$, stopband frequency $\left(\omega_{s}\right)=0.03125 \pi$. The same stopband attenuation, i.e., $100 \mathrm{~dB}$ is taken for model filter as well as for interpolator. The stopband frequency for these filters are $\left(L \omega_{s}\right)=0.0625 \pi$ and $\left(2 \pi / L-\omega_{s}\right)=0.968 \pi$, respectively. The magnitude response of prototype IFIR filter is shown in Figure 5(a) and the magnitude responses of first and last four bands of thirty two-band CMFB is shown in Figure 5(b). The reconstruction error and aliasing error are shown in Figure 5(c) and 5(d), respectively. The optimized value of reconstruction error, aliasing error and reduction in computation cost and group delay are given in Table $\mathbf{1}$.

Example 4: In this example, the performance of proposed technique is compared with the reported work of Furado et al. [9]. Same input parameters as reported are taken for comparison purposes. The obtained error parameters from the proposed technique are given in Table 2. The plot of reconstruction error and aliasing error are given in Figure 6.

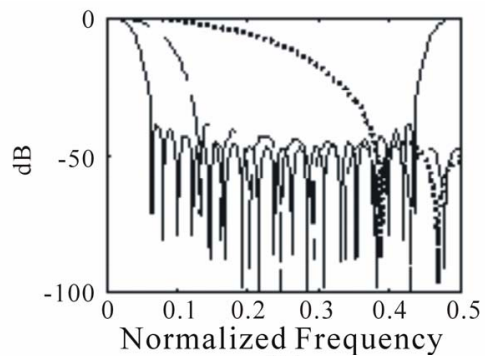

(b)

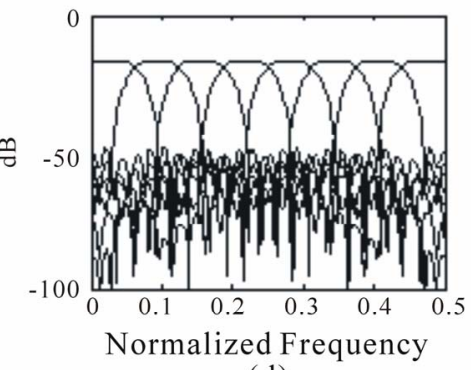

(d)

Figure 2. (a) FIR lowpass filter, (b) (-) Up-sampled model,(- - - -)model filter,(--------) Interpolator filter, (c) IFIR prototype lowpass filter, (d) Eight-band CMF bank.
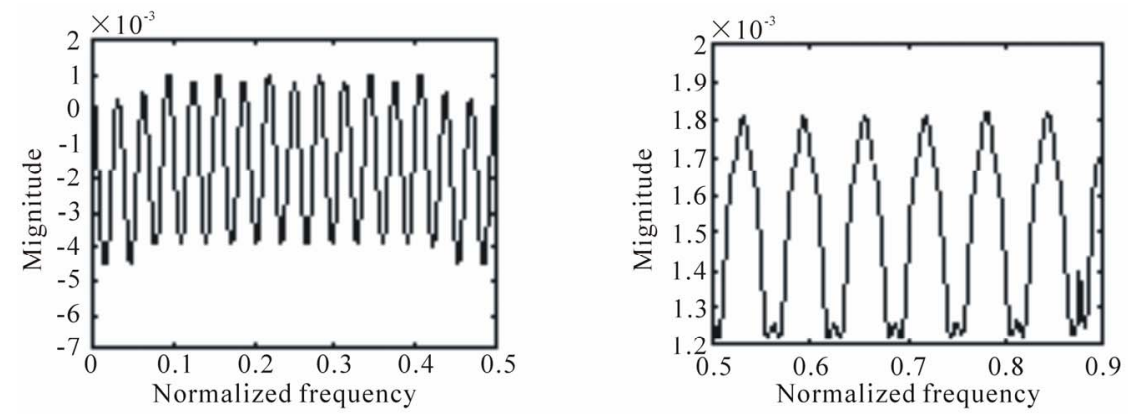

Figure 3. Magnitude response of (a) Reconstruction error, (b) Aliasing error for eight-band filterbank. 


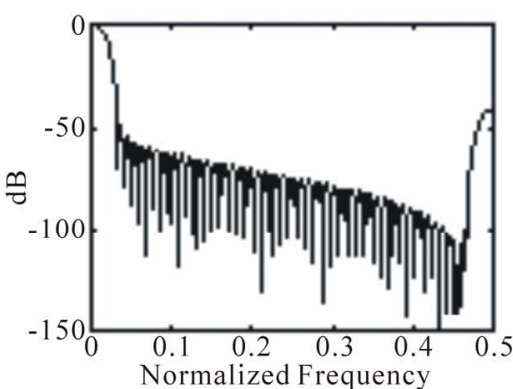

(a)

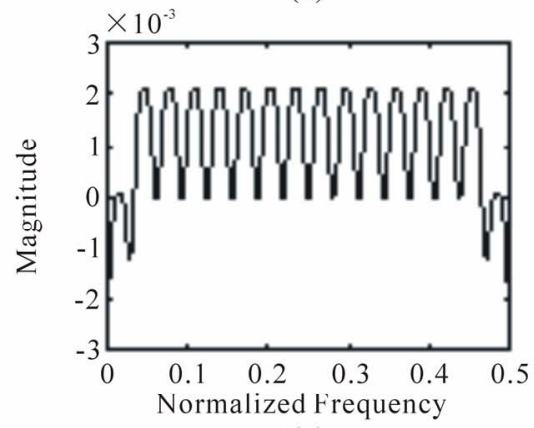

(c)

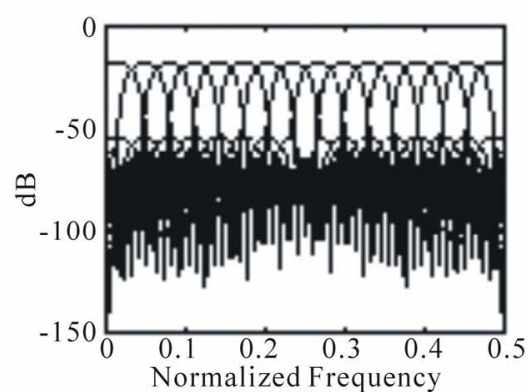

(b)

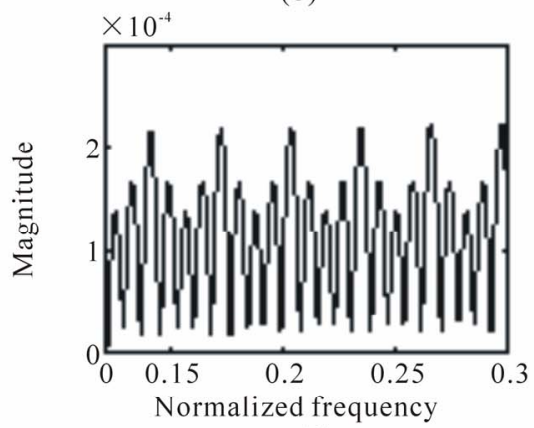

(d)

Figure 4. Magnitude response of (a) IFIR prototype filter, (b) Sixteen-bands CMF bank(c) Reconstruction error, (d) Aliasing error.

Table 1. Performance comparison.

\begin{tabular}{|c|c|c|c|c|c|c|c|c|c|c|c|c|c|c|}
\hline \multirow{2}{*}{ Work } & \multirow{2}{*}{ Approach } & \multirow{2}{*}{$\begin{array}{c}A_{s} \\
\mathrm{~dB}\end{array}$} & \multirow{2}{*}{$M$} & \multirow{2}{*}{$\omega_{s}$} & \multicolumn{5}{|c|}{ Filter order } & \multicolumn{4}{|c|}{$\begin{array}{c}\text { Arithmetic } \\
\text { elements }\end{array}$} & \multirow[t]{2}{*}{$\begin{array}{c}\% \text { Computa- } \\
\text { tional } \\
\text { saving }\end{array}$} \\
\hline & & & & & $N$ & $N_{m}$ & $N_{i}$ & $E_{p p}$ & $E_{a}$ & $\mathrm{E}_{\mathrm{s}}$ & $\begin{array}{l}\text { Ad- } \\
\text { ders }\end{array}$ & $\begin{array}{l}\text { Mult- } \\
\text { liers }\end{array}$ & $\tau_{g}$ & \\
\hline \multirow{6}{*}{$\begin{array}{l}\text { Kha et } \\
\text { al.[10] }\end{array}$} & FIR & 35.8 & 8 & $0.12 \pi$ & 40 & - & - & $5.50 e-3$ & $2.47 \mathrm{e}-3$ & $1.18 \mathrm{e}-2$ & 40 & 20 & 20 & - \\
\hline & FIR & 45.0 & 16 & $0.059 \pi$ & 102 & - & - & $5.95 \mathrm{e}-3$ & $3.89 \mathrm{e}-4$ & $9.00 \mathrm{e}-4$ & 102 & 51 & 51 & - \\
\hline & FIR & 100 & 32 & $0.031 \pi$ & 466 & - & - & $9.12 \mathrm{e}-4$ & $2.38 \mathrm{e}-7$ & $9.9 \mathrm{e}-10$ & 466 & 233 & 233 & \\
\hline & \multirow{3}{*}{ Polyphase } & 35.8 & 8 & & 40 & - & - & & & & 20 & 20 & & \\
\hline & & 45.0 & 16 & & 102 & - & - & & & & 51 & 51 & & \\
\hline & & 100 & 32 & & 466 & - & - & & & & 233 & 233 & & \\
\hline \multirow{3}{*}{$\begin{array}{l}\text { Pro- } \\
\text { posed }\end{array}$} & \multirow{3}{*}{ IFIR } & 35.8 & 8 & $0.12 \pi$ & - & 20 & 06 & $5.46 \mathrm{e}-3$ & $1.41 \mathrm{e}-3$ & $1.0 \mathrm{e}-2$ & 26 & 13 & 13 & 35 \\
\hline & & 45.8 & 16 & $0.059 \pi$ & - & 46 & 06 & $2.1 \mathrm{e}-3$ & $2.62 \mathrm{e}-4$ & $1.0 \mathrm{e}-3$ & 52 & 26 & 26 & 49 \\
\hline & & 100 & 32 & $0.031 \pi$ & - & 267 & 15 & $3.3 e-3$ & $1.80 \mathrm{e}-7$ & $8.8 \mathrm{e}-10$ & 282 & 141 & 141 & 39 \\
\hline
\end{tabular}




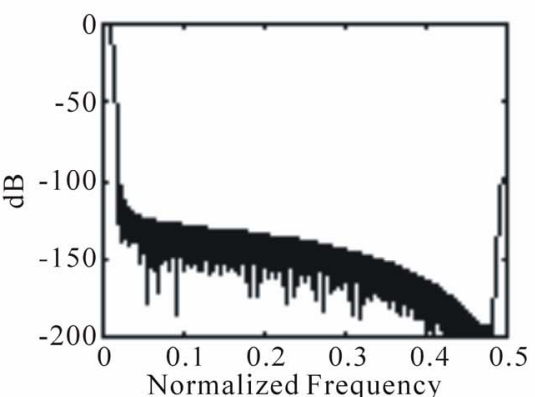

(a)

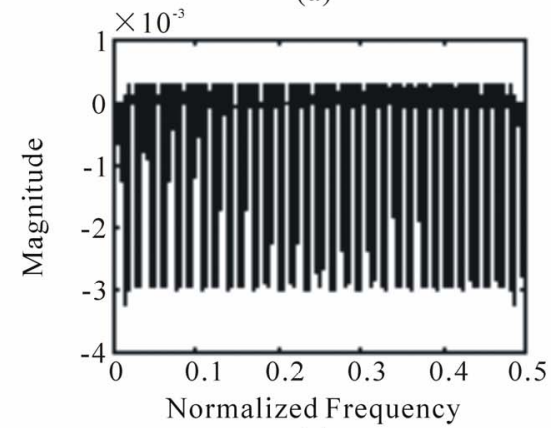

(c)

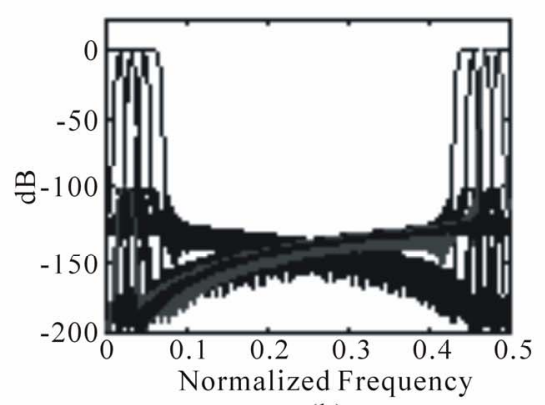

(b)

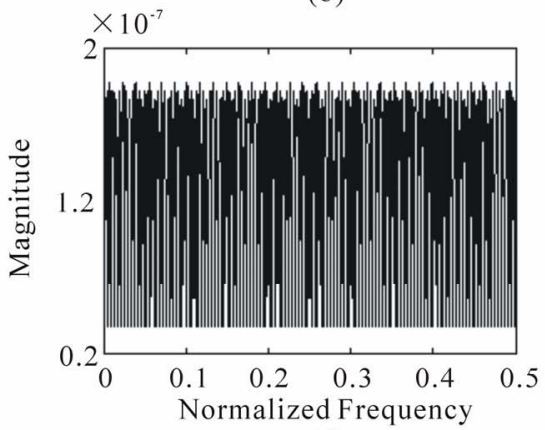

(d)

Figure 5. Magnitude response of (a) IFIR prototype filter, (b) Thirty two-band CMF bank (c) Reconstruction error, (d) aliasing error.

Table 2. Performance comparison with Furado et al. [9].

\begin{tabular}{cccccccccc}
\hline work & Approach & $A_{s}$ & $M$ & $\omega_{s}$ & $N$ & $N_{m}$ & $N_{i}$ & $E_{p p}$ & $E_{a}$ \\
\hline Furado et al. [9] & FRM & 60 & 8 & $0.035 \pi$ & 639 & - & - & - & $2.1 \mathrm{e}-4$ \\
Proposed & IFIR & 60 & 8 & $0.035 \pi$ & - & 122 & 08 & $9.1 \mathrm{e}-3$ & $0.92 \mathrm{e}-5$ \\
\hline
\end{tabular}

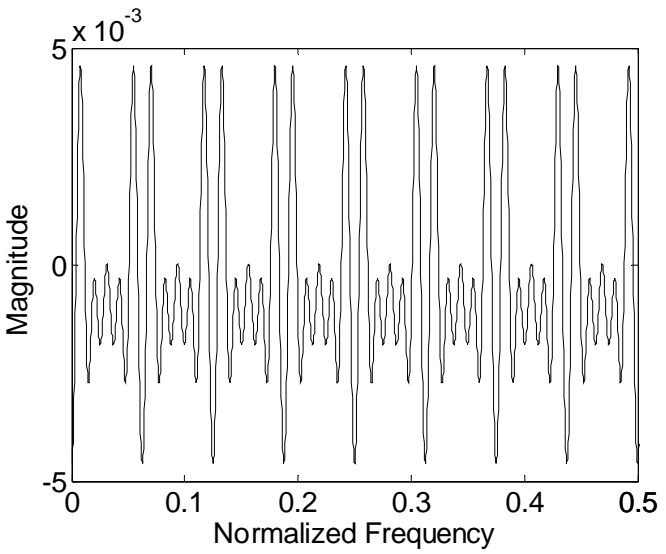

(a)

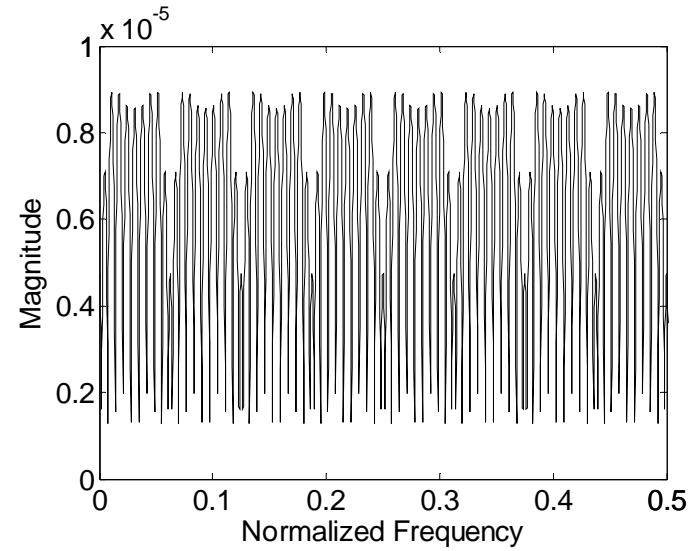

(b)

Figure 6. Plot of magnitude response of (a) reconstruction error (b) aliasing error.

\section{Discussion}

In last two decades, lot of cost effective techniques have been developed for the design of the filterbank. IFIR approach is one of the techniques, which provides significant reduction in computational cost. In this proposed work, the reduction in computation is achieved by using stretch factor of two and better results are obtained. 
In the first example, 8-bands CMFB is designed. The values of resulting reconstruction and aliasing error in Kha et al. [10] and proposed work are $5.508 \times 10^{-3}$; $2.477 \times 10^{-3}$ and $5.46 \times 10^{-3} ; 1.4 \times 10^{-3}$ respectively. Hence, the proposed method offers much better performance in terms of reconstruction and aliasing errors. Apart from lower values of error parameters the proposed method provides reduction in stopband energy, less group delay and $35 \%$ reduction in computational cost in contrast to Kha et al.

Similarly, in second example, 16-band CMFB is designed. It is quite clear from Table 1 that the value of error parameters in the proposed work is much smaller than the Kha et al. [10] with $49 \%$ reduction in computational cost. In third example, 32-band CMFB is designed with the same input parameters as of Kha et al. [10]. As it is clear from the results given in Table 1 that the proposed technique provides smaller value of aliasing error then reported work with reduction in computational cost in terms of multipliers.

The fourth example is quoted for the comparison with the work of Furado et al. [9]. In this approach the prototype filter is designed using FRM technique and the required number of filter order is very large, therefore computational complexity is also high. However, the proposed IFIR technique provides smaller value of aliasing error using much lower value of filter order.

The results in Table 1 indicate that the proposed method provides better performance in terms of computational complexity then the equivalent polyphase implementation. It is also clear from the magnitude response of prototype filter that there is improvement in SLFOR, which provides reduction in stopband energy of prototype filter. The proposed method is not only suitable for lower range of stopband attenuation, but also applicable at higher value of stopband attenuations.

\section{Conclusion}

Efficient design for $M$-band NPR CMFBs has been presented. IFIR technique is used to design the prototype filter, which provides significant reduction in computation cost as well as improvement in SLFOR of the prototype filter. The obtained values of reconstruction and aliasing errors are much smaller than the error values in previous publications for wide range of stopband attenuation. Therefore, the proposed method is useful in variety of applications such as audio and image compression. The improvement in SLFOR can be utilized for better suppression of cross-talk and echo cancellation. The improvement in proposed approach is possible by using multistage IFIR prototype filter.

\section{REFERENCES}

[1] W. P. Zhu, M. O. Ahamed and M. N. S. Swamy, "An Efficient Approach for the Design of Nearly Perfect Reconstruction QMF Banks," IEEE Transactions on Circuits and System II: Analog and Digital Signal Processing, Vol. 45, No. 8, August 1998, pp. 1161-1165.

[2] Y. J. Chen and K. S. Amaratunga, "M-Channel Lifting Factorization of Perfect Reconstruction Filter Banks and Reversible M-Band Wavelet Transforms," IEEE Transactions on Circuits and System II: Analog and Digital Signal Processing, Vol. 50, No. 12, December 2003, pp. 963-976.

[3] C. K. Goh and Y. C. Lim, "Novel Approach for the Design of Two Channel Perfect Reconstruction Linear Phase Fir Filter Banks," IEEE Transactions on Circuits and System II: Analog and Digital Signal Processing, Vol. 45, No. 8, August 1998, pp. 1141-1146.

[4] P. P. Vaidyanathan, "Multirate Systems and Filter Banks," Prentice-Hall, Englewood Cliffs, New Jersey, 1993.

[5] A. Jain, R.Saxena and S. C. Saxena, "An Improved and Simplified Design of Cosine Modulated Pseudo-QMF Filterbanks," Digital Signal Processing, Vol. 16, No. 3, May 2006, pp. 225-232.

[6] T. Q. Nguyen, "Near-Perfect-Reconstruction Pseudo-QMF Banks," IEEE Transactions on Signal Processing, Vol. 42, No. 1, January 1994, pp. 65-76.

[7] T. Q. Nguyen, "Digital Filterbank Design Quadratic-Constrained Formulation," IEEE Transactions on Signal Processing, Vol. 43, No. 9, September 1995, pp. 21032108.

[8] Y. Neuvo, C. Y. Dong and S. K. Mitra, "Interpolated Finite Impulse Response Filters," IEEE Transactions on Acoust, Speech, Signal Processing, Vol. ASSP-32, June 1984, pp. 563-570.

[9] M. B. Furado, P. S. R. Diniz, S. L. Netto and T. Saramaki, "On the Design of High-Complexity Cosine-Modulated Transmultiplexers Based on the Frequency-Response Masking Approach," IEEE Transactions on Circuits and System, Vol. 52, No. 11, November 2005, pp. 2413-2426.

[10] H. H. Kha, H. D. Tuan and T. Q. Nguyen, "Efficient Design of Cosine-Modulated Filterbanks via Convex Optimization," IEEE Transactions on Signal Processing, Vol. 57, No. 3, March 2009, pp. 966-976.

[11] A. Mehrnia and J. A. Willson, "On Optimal IFIR Filter Design," IEEE Proceedings of the 2004 International Symposium on Circuits and Systems, Vol. 3, May 2004, pp. 133-136.

[12] S. K. Mitra, "Digital Signal Processing-A computer based approach," Tata McGraw-Hill Publishing Co. Ltd., New Delhi, 1998.

[13] O. P. Sahu, M. K. Soni and I. M. Talwar, "Marquardt Optimization Method to Design Two-Channel Quadrature Mirror Filterbanks," Digital Signal Processing, Vol. 16, No. 6, November 2006, pp. 870-879.

[14] C. D. Creusere and S. K. Mitra, "A Simple Method for Designing High-Quality Prototype Filters for $M$-Band 
Pseudo QMF Banks," IEEE Transactions on Signal Processing, Vol. 43, No. 4, April 1995, pp. 1005-1007.

[15] Y. P. Lin and P. P. Vaidyanathan, "A Kaiser Window Approach for the Design of Prototype Filters of Cosine-Modulated Filterbanks," IEEE Signal Processing
Letters, Vol. 5, No. 6, June 1998, pp. 132-134.

[16] J. D. Johnston, "A Filter Family Designed for Use in Quadrature Mirror Filterbanks," IEEE International Conference on Acoustics, Speech and Signal Processing, Denver, 1980, pp. 291-294.

\section{Appendix}

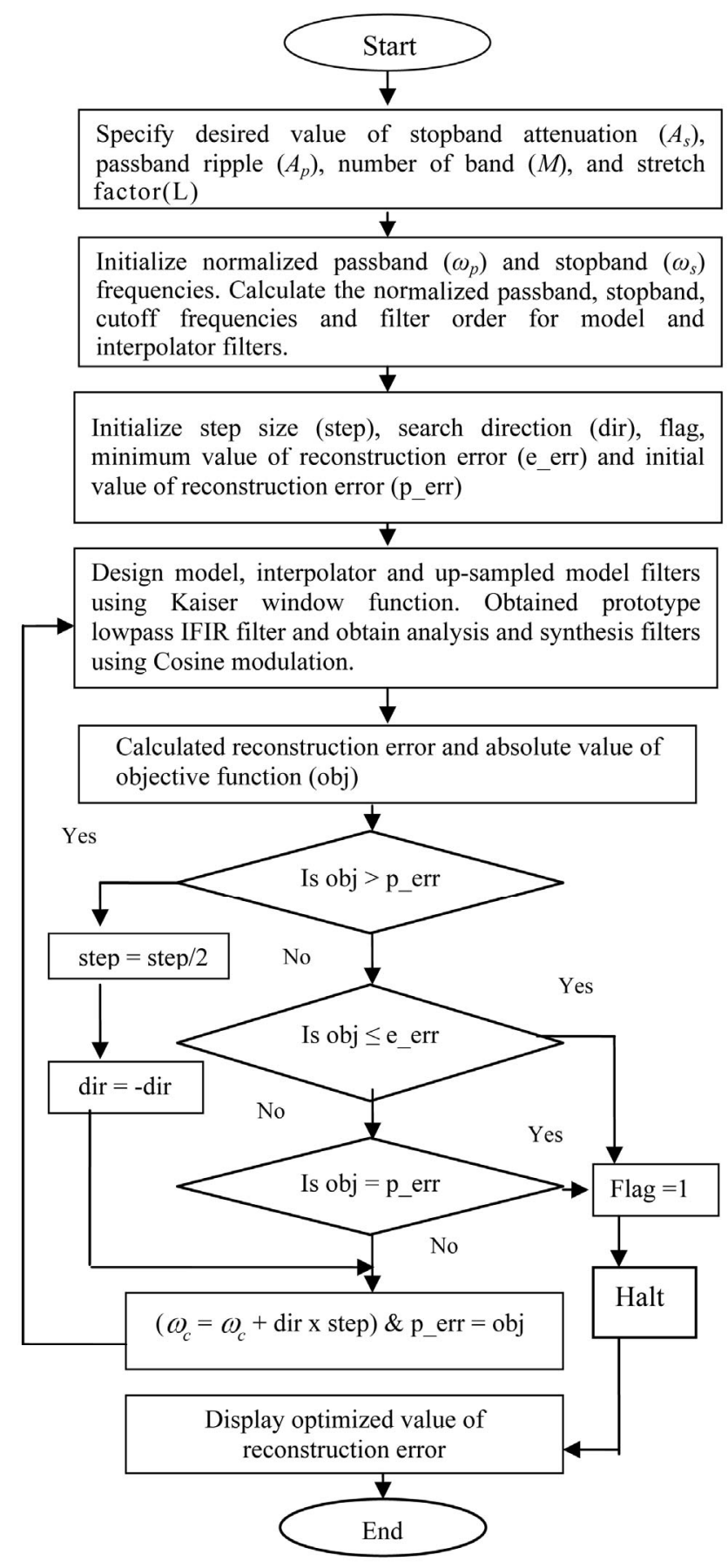

